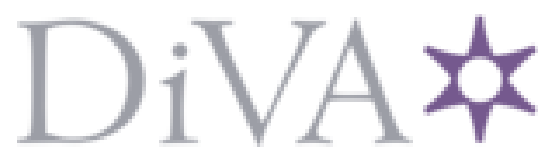

http://www.diva-portal.org

This is the published version of a paper published in First Monday.

Citation for the original published paper (version of record):

Marti, P., Peeters, J., Trotto, A., Tittarelli, M., True, N. et al. (2015)

Embodying culture: Interactive installation on women's rights.

First Monday, 20(4): 6

http://dx.doi.org/10.5210/fm.v20i4.5897

Access to the published version may require subscription.

N.B. When citing this work, cite the original published paper.

Permanent link to this version:

http://urn.kb.se/resolve?urn=urn:nbn:se:umu:diva-115881 


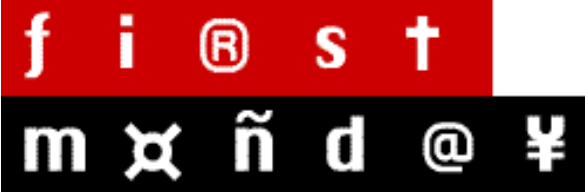

PEER-REVIEWED JOURNAL ON THE INTERNET

\begin{tabular}{|c|c|c|c|c|}
\hline HOME & ABOUT & LOGIN & REGISTER & CURRENT \\
\hline ARCHIVES & AN & NCEMENTS & SUBMISSIONS & \\
\hline
\end{tabular}

OPEN JOURNAL $\underline{\text { SYSTEMS }}$

\section{$\underline{\text { Journal Help }}$}

Home > Volume 20, Number 4 - 6 April 2015 > Marti

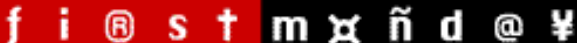

PEER-REVIEWED JOURNAL ON THE INTERNET

Embodying culture: Interactive installation
on women's rights
by Patrizia Marti, Jeroen Peeters, Ambra Trotto,
Michele Tittarelli, Nicholas True, Nigel Papworth,
and Caroline Hummels

\section{Abstract}

The paper describes an interactive installation exploring perspectives on women's rights, triggering visitors' personal reflections through an immersive experience. Starting from the life histories of the women depicted in three paintings from fifteenth, sixteenth and seventeenth century, we explored three themes: emancipation, self-determination and violence. In the installation, representations of these three paintings were fragmented into panels, floating in the space suspended from a selfstanding structure. On these elements, both the original painting and a writhing of visual material were dynamically displayed using a projector. The presence and movement of visitors in the room was tracked by means of a Kinect ${ }^{\mathrm{TM}}$ camera and influenced both the position and movements of the panels. A software crawler monitored discussions and debates on social networks. The intensity of these discussions was reflected in the movements of the panels and the content of the projections. The purpose of this interactive installation is to engage visitors in composing a harmonious picture of the complex domain of women's rights. The experiential form confronts visitors with the opinions of other people debating the theme worldwide. The installation was the outcome of a craft-inspired learning module, grounded on constructivism and reflective practice.

\section{Contents}

Introduction

Conceptual pathway

The space

Interactive installation

Hardware and software

Artwork exhibition room

Backstage

Reflection-in-action

Reflection-on-action: Bridging the real and virtual

\section{USER}

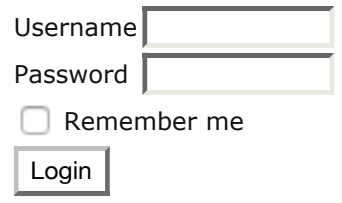

JOURNAL

CONTENT

Search

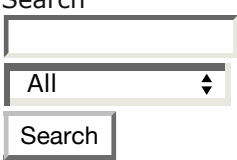

Browse

- By Issue

- By Author

- By Title

- Other Journals

FONT SIZE

CURRENT ISSUE

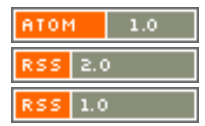

ARTICLE TOOLS

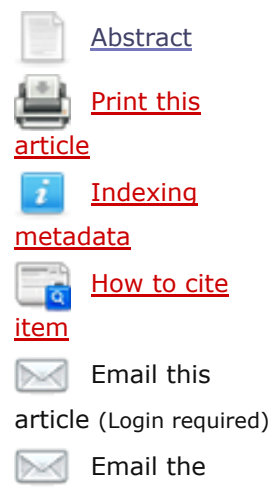

author (Login required)

ABOUT THE AUTHORS

\section{Introduction}

Ballade of Women is an interactive installation that resulted from an intense learning experience. It was developed during the third edition of Light through Culture, an international school of design, founded in Siena in 2011. The school has the objective to provide a highly qualified, multidisciplinary and project-based education to bachelor's, master's and Ph.D. students. The aim of the school is to create a meaningful context for learning, where students can reflect together on socio-cultural issues through design, by building interactive installations (Marti and Overbeeke, 2011).

The school is grounded on the idea that cultural contexts and hands-on activities are important. This is the reason why in Light through Culture, we promote the design of interactive installations. These are built as the
Patrizia Marti University of Siena

Senior Researcher at the Department of Social, Political and Cognitive Science Department,

University of Siena

Jeroen Peeters Umeå University,

Sweden 
product of a highly iterative process, entailing the repetitive transition from abstract to concrete, and back. This process enables a gradual synthesis and understanding of limitations and potentials of intermediate solutions, and of the complex relationship between the design act, the contents, and fabrication approaches to achieve a desired outcome (Marti and Overbeeke, 2011).

Light through Culture combines different approaches and learning theories, with the intent of producing new knowledge, artefacts, and practices that account for and potentially impact learning and teaching. In Light through Culture, knowledge is acquired through the exercise of craftinspired learning practices, throughout design and fabrication processes. Learning through design offers several benefits: tangible outcomes enable the participants to reflect on the role of design in a changing socio-cultural context, and these prototypes are validated through use. More specifically, the vision of Light through Culture is closely connected to constructivism (Birenbaum, 2003), reflective practice (Schön, 1983) and craftsmanship (Sennett, 2008).

There are, of course, different ways in which these approaches can be put into practice. For example, it is not obvious what a constructivist learning environment should look like and how meaning construction can be facilitated in the most effective way.

The constructivist learning environment of Light through Culture is a rich socio-cultural environment (usually a museum or historical site) where learners work together. Through practice, they draw upon contents using a variety of tools, building and experimenting with prototypes and supporting each other in their pursuit of learning goals. They have the opportunity to plan, organize, monitor and improve their products in a collaborative way. In this environment students are challenged by authentic tasks and complex problems (dealing with profound themes that have a social and societal relevance), and have the opportunity to reflect on the process and outcomes of their activity by presenting their projects to be experienced by the public.

Such reflective practice can take shape in different forms and modalities. Dewey (1933) distinguished between 'routine action' and 'reflective action': routine action is guided by factors such as authority and institutional definitions and expectations. Reflective action implies a willingness to engage in constant self-appraisal, improvement and development. It entails flexibility, analysis and social awareness (Pollard, 2005). Schön (1983) states that reflection-in-action concerns thinking about something whilst being engaged in doing it, having a feeling about something and practicing according to that feeling.

This view of the reflective practice is close to Sennett's concept of craftsmanship (2008). Students learn through doing and reflect on their actions. As crafts persons, they have a desire to do good work for its own sake; they trust their senses, intuition, imagination and skills to make and reciprocally to think, meanwhile exploring ambiguity, complexity, resistance and failure.

The third edition of Light through Culture leveraged on craft-inspired and reflective learning to stimulate awareness of women's rights. In particular, the project faced the themes of violence, self-determination and emancipation. Such themes are inherently complex, affording many different points of view. The installation merged embodied and situated perspectives of visitors (off-line) with virtual (online) perspectives expressed by people active on social media. By weaving views from both the physical and digital domain, the installation explored how these different domains might influence the dynamic information landscape created by the exhibition. In this way, we respond in a designerly way to the quest for uncovering how "particular material properties of digital technology affect the (un)making of [normative views of gender]" [1]

In the third edition of Light through Culture, we focused on the opportunity for design to catalyse a cultural shift concerning social awareness and learning practices. This was approached in two ways: Firstly by encouraging the application of a design process where learning is based on skills in craft approach. And secondly, by providing an assignment with an explicit cultural focus, in this case the design of an installation that encourages the reflection on women's rights by eliciting a direct bodily engagement of visitors. Such bodily engagement induces the occurrence of an experience for the visitor. A direct experience entails a constitution of thoughts, judgment and opinions in the visitor: she can't escape it.

Ph.D. and master's students, as well as faculties from the Interactive Institute Swedish ICT, the University of Siena and the Eindhoven University of Technology joined the project. The interactive installation was developed in partnership with Fondazione Monte dei Paschi di Siena and Vernice Progetti Culturali.
Interactive Institute Swedish ICT and Department of Informatics, Umeå University, Sweden

Ambra Trotto

Umeå School of

Architecture, Sweden

Interactive Institute Swedish ICT and

Umeå School of

Architecture, Sweden

Michele Tittarelli

University of Siena,

Italy

Department of

Social, Political,

Cognitive Science,

University of Siena,

Italy

Nicholas True

Umeå University,

Sweden

Department o Informatics, Umeå

University, Sweden

Nigel Papworth Interactive Institute

Swedish ICT,

Sweden

Caroline Hummels Eindhoven University of Technology, the Netherlands

Department o Industrial Design, Eindhoven University of Technology, the Netherlands

\section{Conceptual pathway}

The design team was given the opportunity to browse the private art 
collection of the Fondazione Monte dei Paschi di Siena. Three paintings were selected as the starting point of the design of the exhibition. These paintings illustrate three historical characters and were chosen to reflect the three themes that eventually informed the narrative of the exhibition.

In the introduction to their book, Deviant women, Tiina Mäntymäki, et al. (2015) explore how women portrayed in cultural expressions, who clearly deviate from the accepted social norms, have influenced feminist thought. The sociological notion of Deviance implies that there is a collective perception of difference that members of a community consider somehow morally offensive. Inspired by such notion, the design team used this common thread amongst the three characters as a conceptual driving force for their design approach:

\begin{abstract}
Cleopatra (by Marco Pino, sixteenth century).
Cleopatra was a Greek queen of Egypt, the last in a line stretching back to Alexander the Great. Hounded and wooed by the Roman Empire for her position and power, she was known for her seductive skills and her ravaging intelligence. This painting was used to support our narration on the theme of emancipation.
\end{abstract}

Maria Maddalena (by Rutilio Manetti, seventeenth century). Maria Magdalena was vilified by early Christian history, which confused facts and impeded her to be remembered as an honest human and to be acknowledged as the symbol of knowledge in the Gospels. This painting was used to support our narration on the theme of selfdetermination.

Lucia di Siracusa (by Maestro dell'Osservanza, fifteenth century). Lucia di Siracusa was denounced to the Roman police and prosecuted for her religious beliefs as a revenge for having refused a pretender. She was deprived of her free will, her dignity and condemned to a painful and denigrating death. This painting was used to support our narration on the theme of violence and torture.

A common thread amongst these three characters is that they embody the notion of deviance: many versions of their stories distort their image. Such versions were produced by the accepted moral of the communities in which such women were living. Information is multiple and ambiguous, at times even controversial. At the same time, women's rights are a complex matter: while respecting the diversity of the individual, they sanction their universal validity. This paradox shows their fragility. The way the three chosen themes are treated in media, social media and interpreted are infinite and depend on so many factors that make it impossible to easily formulate a permanent safe opinion or stance.

These reflections led the design team to focus on fragmentation as the natural concept to explore. Fragmentation became the aesthetic drive to design the experience of Ballade of Women. Thus, by embracing fragmented visuals, audio and online content, the design team could create an installation that allowed visitors to raise questions appropriate to these themes. Furthermore, it embodied and respected the complexity inherent to such themes.

The installation was a dynamic space constituted by floating and mobile panels, created by slicing digital versions of the paintings along their contours. The behaviour of the panels was influenced by the physical presence of the observer as well as by the virtual presence of online discussion groups debating the themes of the exhibition. When the panels were positioned in a fragmented way, they displayed media collected from online groups, discussing the issues addressed in the exhibition. Software continuously monitored news related to the treated themes and online discussions generated by this news. The number of threads was an input for the installation and influenced the speed of movements as well as dynamics with which the informative content was displayed in the projections. The soundscape allowed the viewer to pick up fragments of poems, related to the three themes. Verses were whispered and vanished immediately after being heard. The dynamics of the fragmented panels redefined the contours of the three paintings from specific viewpoints in the space. By looking at three paintings, in a continuously changing manner, the installation aimed at playing with and perhaps changing our perspective of these universal issues, tackled from a feminine perspective.

The experience was designed to trigger personal reflections in visitors by actively involving them in the exhibition. The goal was to suggest that each of us could contribute to compose a coherent picture of women's rights, by approaching it, and by being confronted with points of view of other people, facing the same topics from different perspectives from all over the world. 


\section{The space}

The exhibition developed along five spaces (Figure 1): the Entrance; the Introduction Space; the Interactive Installation; the Artwork Exhibition Room; and the Backstage room.

(1) Entrance

(2) Introduction space

(3) Interactive Installation

(4) Artwork Exhibition Room

(5) Backstage

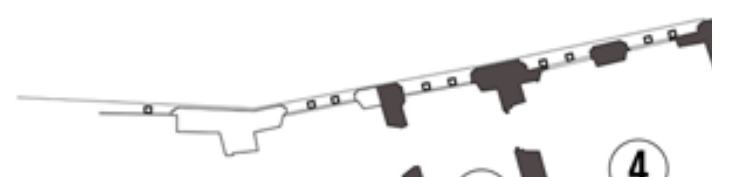

Figure 1: The exhibition.

\section{Interactive installation}

\section{Architectural components}

The installation was composed of a large freestanding and triangular aluminium frame flanked by three independent triangular columns (Figure 2).

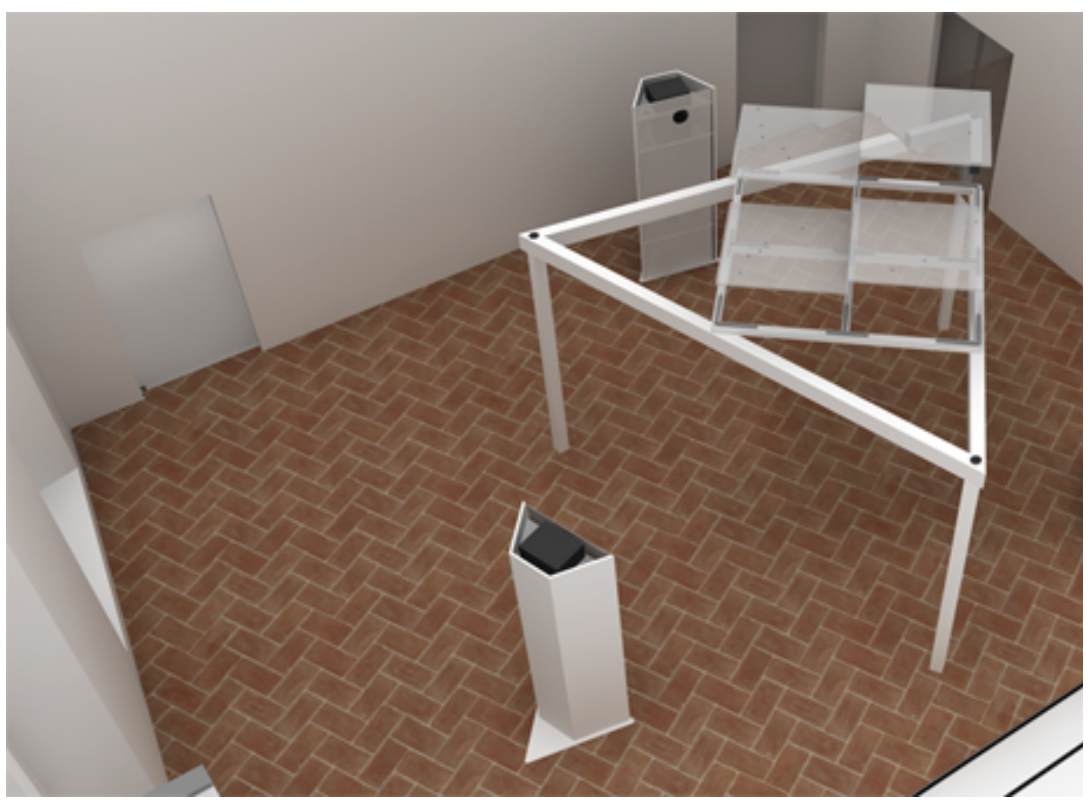

Figure 2: Interactive installation. 
The aluminium frame was custom fabricated for the installation by Modelleria Ferrieri s.r.I. The top of the frame was machined to support large acrylic sheets on which the motors providing movement to the panels were mounted. Laser cut acrylic components were fabricated to serve as gears for the motor to control the movement and timing of the panels. White, opaque acrylic was laser cut to form the painting fragment panels. These panels were then suspended with nylon wire from the acrylic supports attached to the motors.

\section{Painting panels}

Using Maya 3D-modelling software, the three paintings were fragmented into several panels, based on the contours of the visual elements present in the original paintings. This resulted in a total of 16 panels that were placed in a 3D space in a staggered manner. Each of the panels was scaled in order to adjust for perspective deformation using the same software. The panels were laser cut from thin white acrylic, and attached to the motors by means of clear nylon wire. Projectors mounted in the three pylons that surrounded the main structure, projected a high-quality digital image of the whole painting onto the separate acrylic panels. The result was a fragmented and three-dimensional view of the three paintings, suspended from the main structure.

\section{Tertiary columns}

Three triangular structures were fabricated utilizing lightweight particleboard (Figure 3 ). The front of the columns was covered using white fabric. These structures were placed parallel to each side of the triangular main structure. Within these columns, projectors and other necessary technologies were housed that needed to be hidden from participants to maintain the desired aesthetics of the installation.

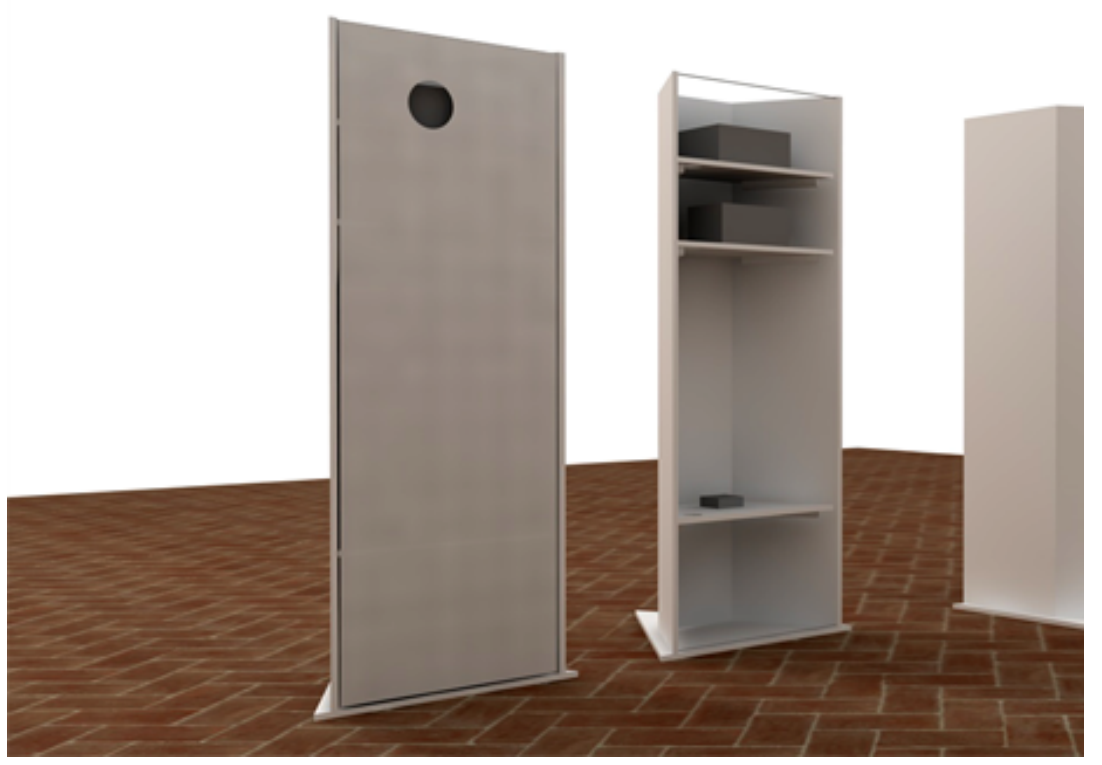

Figure 3: Tertiary columns. 
(1) Hanging panels

(2) Mounting panels for the motors and Arduino micro-controllers

(3) Aluminium frame

(4) Pillars with computers, projectors directional speakers, and the channels to carry the connecting cables. Also they each had an LED light anray

(5) Kinect Cameras

(6) Trigger zone for each Kinect Camera

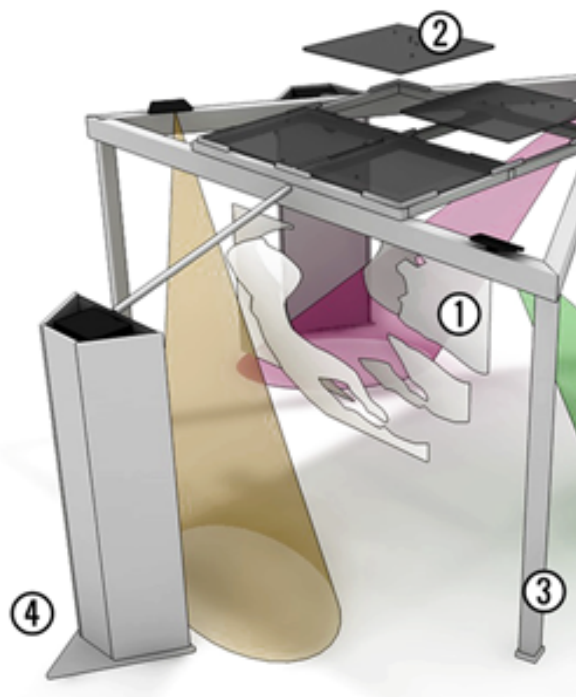

Figure 4: A schematic representation of the installation's com

\section{Hardware and software}

Hardware components

The hardware components included: three projectors, three sets of laser cut acrylic panels (one for each painting), three Arduino microcontrollers, three sets of stepper motors, three Microsoft Kinect ${ }^{\mathrm{TM}}$ cameras, three LED lights, and three Intel NUC (Next Unit of Computing ${ }^{\mathrm{TM}}$ ) mini computers running Microsoft Windows $7^{\mathrm{TM}}$.

\section{Hardware implementation}

The installation was created as three sets of hardware functioning independently to create a cohesive whole. Each of the three computers ran software that controlled one set of motors that moved a set of panels. Kinect cameras were employed to identify and track participants, when one person was "locked onto" while standing in a pre-defined "sweet spot" the panels would align to briefly reveal the painting to that viewer.

Projectors in each column projected the images onto the panels. LED lights were positioned on the top of the triangular structure and programmed to cycle through and project dominant colours from the paintings to add to the immersive experience.

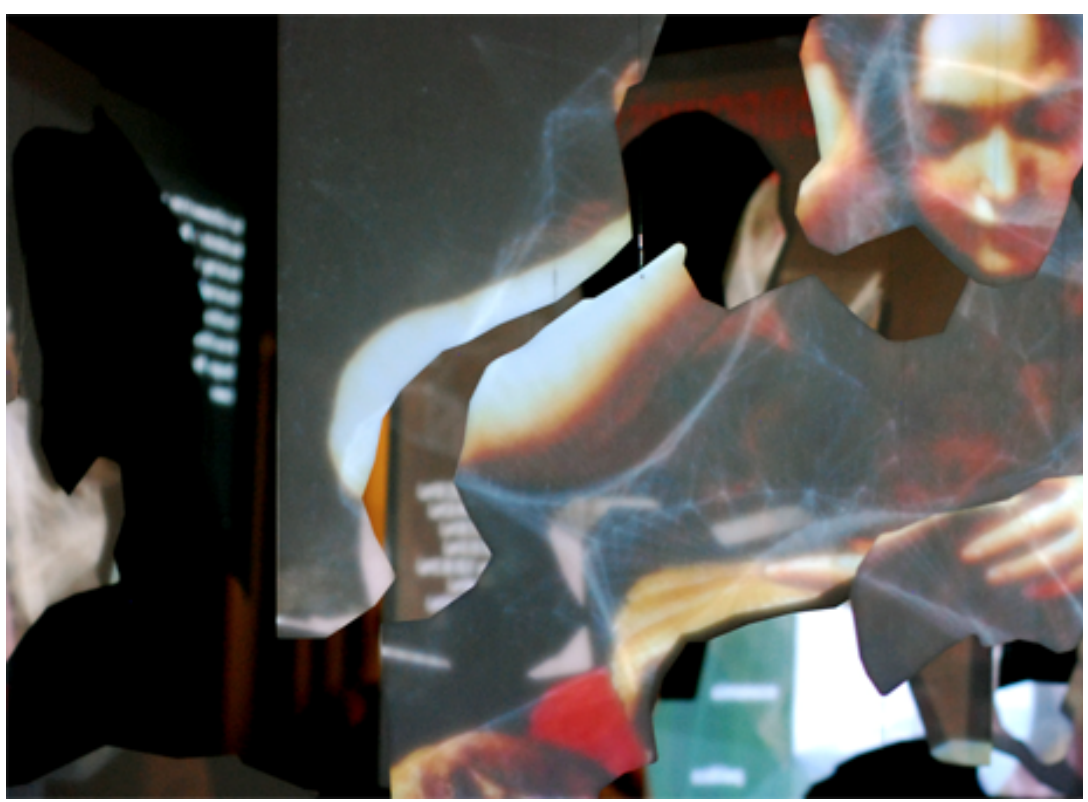

Figure 5: An image of the fragmented panels of the Maria Maddalena, viewed fr 
spot".

The movements of the panels, as well as the visual contents, were influenced not only by the physical presence of the observers detected by the Kinect cameras, but also by the opinions expressed through social networks concerning women's rights. WhatsOn is a software crawler developed by QuestIt, which performed a continuous monitoring of news regarding the topics of self-determination, emancipation and violence, and the way they were being discussed through online posts and debates. The number of discussions generated on Internet was an input to the structure, which determined both its movement and visual contents. The movement of the panels was sensitive to online discussions, quotes and social media sources which were also overlaid onto the projected paintings (Fiqure 5).

\section{Audio components}

Four directional speakers were employed, each with a MP3 player as a source. Two directional speakers were installed in columns and the other two were attached directly to the aluminium frame. Directional speakers differ from conventional speakers: With a standard speaker, the sound spreads out in a cone (Figure 6). Usually the signal bounces off all surfaces, and during its path the sound travels and spreads loosing force, which is experienced as a change in volume. With a directional speaker, two ultra-high, sonic signals are sent. These travel parallel to each other with very little loss of signal strength. When they hit an object (a wall, panel, human being, etc.) the difference between the two signals creates a sound source based on the information in the two ultra-high, sonic signals. The listener identifies this point of impact as the source.

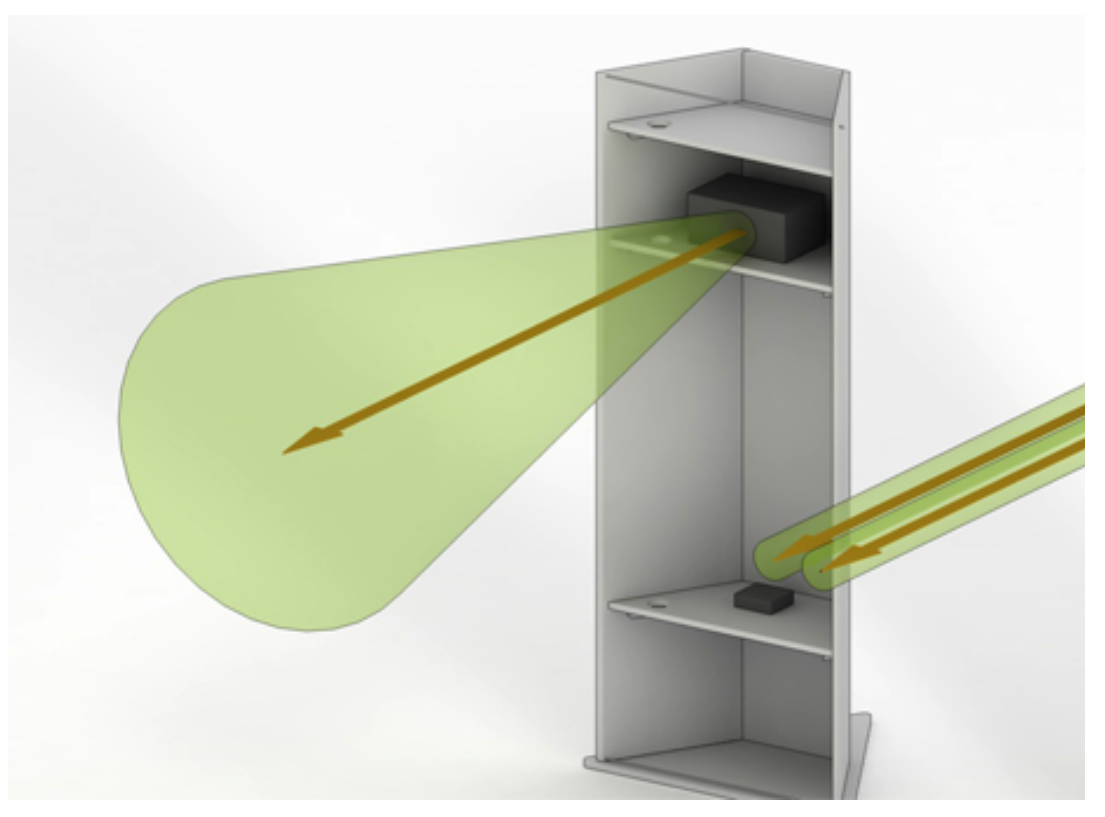

Figure 6: Schematic showing the difference between audio waves coming from a a directional speaker (right).

\section{Audio implementation}

The design direction around fragmentation was supplemented by fragmented audio, to create a immersive environment. Utilizing the unique properties of directional speakers to bounce the narrow beamed sound signal off the rotating panels, it was possible to create sound that moved around the room. The sound projected in this way gave an ethereal and obscured experience. The directional speakers produced sound in such a way that it seemed the sound was broadcasted directly into the listeners' head, specifically for them. The sound consisted of a selection of poems read, almost whispered, in different languages [2] ]. The poems were selected for their relevance to the three themes of the exhibition. 


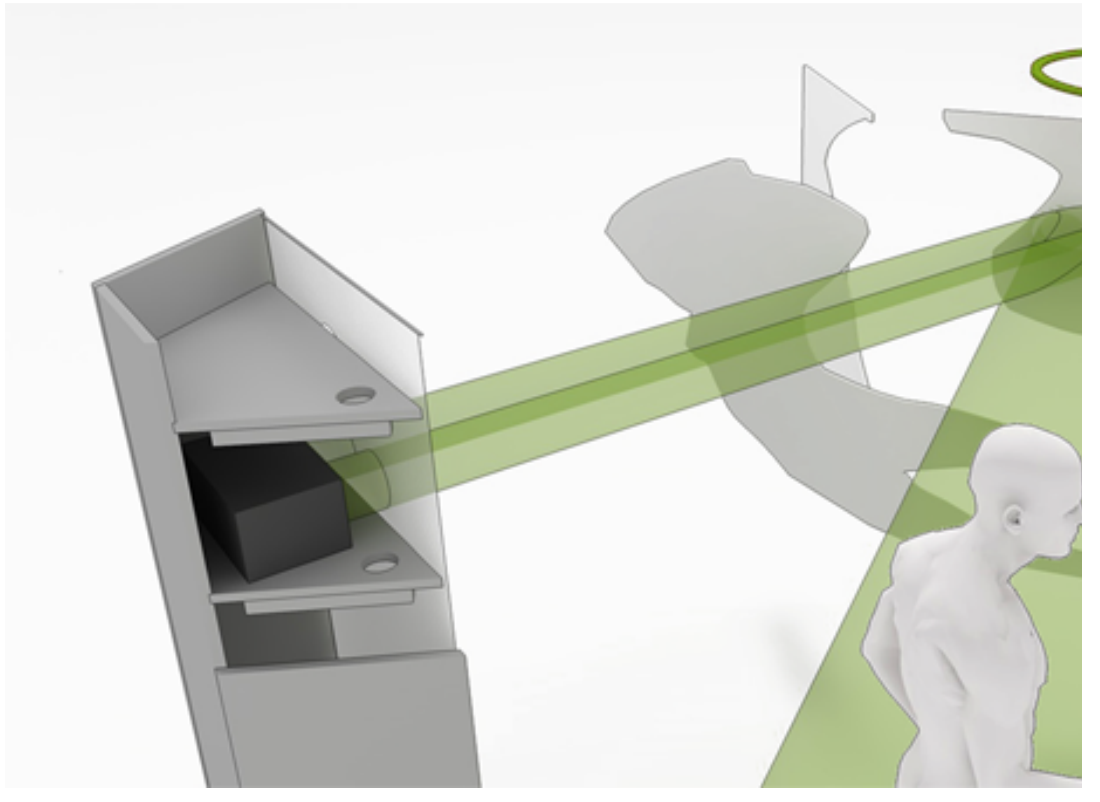

Figure 7: The directional speakers are aimed at the rotating panels, created a around the room, passing visitors and creating a fragmented experience of

Two additional sound shower directional speakers were mounted on the aluminium frame. The first one projected sound toward the entrance of the Interactive Installation space. The sound developed along the stairs getting to the room, to prepare the visitors to the experience. The second sound shower speaker was oriented toward the entrance of the next space, the Artwork Exhibition Room, to guide people to continue the visit. These directional speakers delivered high-quality, focused audio to the desired areas, without disturbing the surroundings. The sound consisted of original music composed for the exhibition.

\section{Artwork exhibition room}

As anticipated in the introduction, the exhibition room contained three precious paintings of the private collection of the Fondazione Monte dei Paschi di Siena: Cleopatra, Maria Maddalena and Lucia of Siracusa http://www.pinterest.com/balladeofwomen/paintings/ - text and images provided by Fondazione Monte dei Paschi di Siena and Vernice Progetti Culturali s.r.l.

These paintings are part of a private collection. Light through Culture contributed to disclose these masterpieces and make them visible to the public.

\section{Backstage}

This room recreated a sort of "directing room", visualizing the discussions being generated on Internet in real-time, and simulating their impact on the main Interactive Installation. In this room, visitors could experiment with the technologies used to build the main structure. A small-scale model of the main installation was built, using a transparent acrylic cube. Three servos were mounted on top of the cube and moved three stylized images of the original paintings showed in the exhibition (Figure 8). 


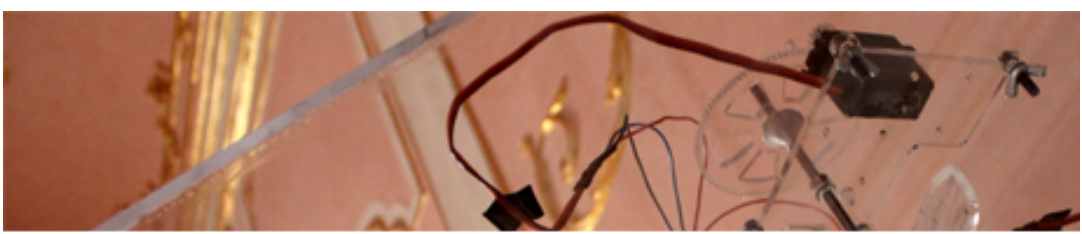

Figure 8: Small scale simulator.

The software crawler WhatsOn was connected to an Arduino board. The tweets related to the themes of emancipation, self-determination and violence, detected by the crawler using keyword searches, were used to move the servomotors and therefore the images attached to them.

The tweets were detected approximately every minute. The theme that gathered the biggest number of new posts among the first five results, determined a slight movement of one of the servos. In this way, the visitors could send a tweet and experience the impact of their opinion on the structure in real time (Figure 9).

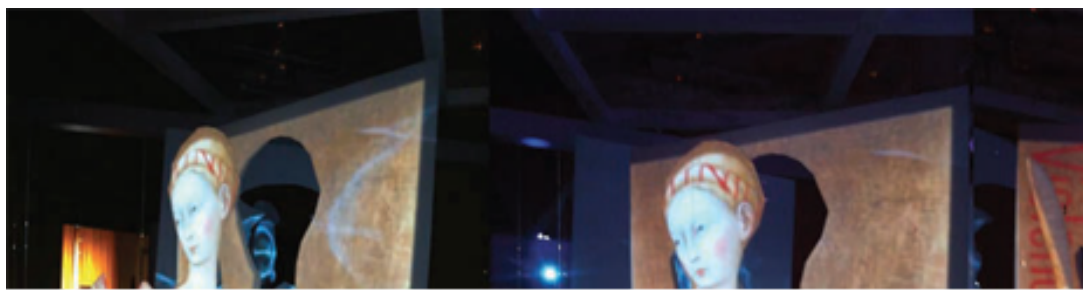

Figure 9: A sequence showing the Lucia di Siracusa panels line up from a pointspot". The image on the right shows an example of data sourced from the Int painting. In this case, the graphical overlay displays a statement sourced from crawler.

\section{Reflection-in-action}

Developing the interactive installation was not the end of the learning process, but a step forward in the reflective practice. Schön (1983) defines two types of reflection: reflection-in-action and reflection-onaction. Reflection-in-action happened whilst we were working/prototyping and it allowed us to reshape our design. Students had to think things through, in particular during the iterative refinement of a prototype. This helped them find a viable solution through "trial-and-error". 
Reflecting-in-action was the leitmotiv of the exhibition. It was called forth by unexpected events in the design process, like unanticipated, beautiful choreographies of the panels, or the aesthetics of the installation that appeared as a holistic combination of sound, music, changing shapes, ambiguity of contents or unforeseen interactions among the components of the structure. Each surprise caused us to question how it occurred, how to cope with that and how our thinking needed to change to address this new event.

\section{Reflection-on-action: Bridging the real and virtual}

The second level of reflection is reflection-on action. This occurred after the design was completed, and the exhibition was open to the public from the 18 June to 2 July 2013. This level of reflection was achieved by thinking back to our experiences, testing our existing beliefs and questioning decisions and results. Doing so, we developed new questions and ideas, and generated new knowledge.

An example of reflection-on-action carried out after the opening of the exhibition, was the observation of the interplay between the moving panels and the contents generated by the social media. Since the installation was dynamic, new contents and patterns of movements were generated every day. For example, in the time frame of the exhibition, the Italian astrophysicist Margherita Hack (1922-2013) died. She gained fame as a science writer able to explain her research in easy-to-understand language and also for being the first woman to lead an astronomical observatory in Italy. In her memory, social networks mentioned her as an example of feminine self-determination. This was reflected on the posts projected on the panels depicting Maria Magdalena, our icon of selfdetermination. We observed students and visitors noticed the news and discussed the event, connecting the life of the two women, comparing their conditions in the different historical periods.

Another interesting phenomenon observed during the exhibition was related to the movement of the panels. This behaviour of the installation was influenced by online discussions, quotes and statements sourced from social media. Excerpts of these online discussions or statements were also overlaid onto the projected paintings. This combined embodied interaction with the physical installation and social computing, resonating with views of embodiment (e.g., Dourish, 2001), and meant the installation merged online and off-line as well as past and present thinking and action around the exhibition themes.

For example, news and posts concerning episodes of violence against women were often displayed on, and clearly reflected in the behaviour of, the panels displaying Lucia di Siracusa. A similar, but less evident phenomenon occurred for the theme of emancipation, where the crawler collected images and posts related to the use of the woman's body as a tool of power and seduction. Our observations regarding the behaviour of visitors and the online content suggest that violence and emancipation are widely debated themes on the Internet, whilst the theme of selfdetermination is more controversial, ambiguous and perhaps therefore less discussed on social media.

An example of how current political issues related to women's rights emerged as part of the installation is a discussion around the Istanbul convention, adopted by the Council of Europe Committee of Ministers on 7 April 2011. The convention aims to form policies and measures to counter violence against women. Interestingly, the convention puts emphasis on "domestic violence", in its all declinations of physical, sexual, psychological or economic violence that can occur within the family. This was highlighted and discussed online during the exhibition. On 19 June 2013, news was posted on the Twitter account @BalladeOfWomen reporting that the Italian Senate approved the Istanbul Convention to combat violence against women. The post "the Istanbul Convention is now law of the Italian state" was displayed and overlayed on the digital fragments of the Lucia di Siracusa painting. Similar posts were generated like the one received on 17 June 2013: "The City Council of \#Venezia approves two motions to apply law 194 to ensure the right of self-determination \#women"; or the another, received on 7 June 2013, mentioning a letter written by Lord Avebury and Lord Carlile to U.N. High Commissioner for Human Rights on the critical situation in Bangladesh regarding the violation of women's rights.

The ambiguity of interpretation, elicited by the interactive installation, connected to the ambiguity of information, to its controversial nature. This offered different points of view and suggested the need for clearer understanding and embodiment of these issues. The information flow reflected both the old and new techniques for imparting, sourcing and consuming information.

The purpose of Ballade of Women was to interactively engage visitors, empowering them to constitute their own take and point of view on themes related to gender. By giving it an experiential form, visitors were drawn into a pro-active attitude and confronted with the opinions of other people. Our observations of the visitors' behaviour in the space, online statements posted to social media, as well as the written records of their 
thoughts on the guest book suggest that relevant discussions were successfully raised and our approach to respect the complexity of the theme was well received. F

\section{About the authors}

Patrizia Marti is part-time Professor in the Department of Industrial Design at Eindhoven Technical University. She is Senior Researcher in the Department of Social, Political and Cognitive Science at the University of Siena where she teaches Experience Design. She is Director of the Laboratory of Robotic and Learning Technologies and Rector's delegate for technological innovation in the humanities at the University of Siena. She has been an invited keynote speaker at various international conferences. She has been also the editor for special issues of international journals. Her research activity concerns designing systems facing cultural, aesthetic and social issues through embodied experiences. E-mail: marti [at] unisi [dot] it

Jeroen Peeters studied Industrial Design at the Royal Melbourne Institute of Technology (Australia) and the Eindhoven University of Technology in the Netherlands, receiving his Master's degree (cum laude) in 2012. In 2010, he co-founded the interactive lighting design studio De Bende and worked on several public lighting projects. In 2012, Jeroen joined Interactive Institute Swedish ICT as a Design Researcher and became a Ph.D. candidate in the Department of Informatics at Umeå University in Sweden. In his work, he explores how to design for engagement through aesthetics of interaction. E-mail: jeroen [dot] peeters [at] tii [dot] se

Ambra Trotto is currently studio director in Umeå, and senior lecturer in the Umea School of Architecture. She closely collaborates with the Designing Quality in Interaction group of the Eindhoven University of Technology. She graduated with honors in architecture in April 2003 at the University of Florence (Italy) and defended her doctoral thesis in December 2011 at the Eindhoven University of Technology, Department of Industrial Design, Designing Quality in Interaction Group, where she worked from 2007 to the beginning of 2012. During her research she explored the field of ethics in design for intelligent products and systems, applying processes that focus on the sharing of making in multicultural environments and has contributed to the foundation of the Rights through Making approach.

E-mail: ambra [at] tii [dot] se

Michele Tittarelli is a designer/interaction designer. He graduated with honors and dignity of publication in the Faculty of Industrial Design at the University of Florence. Since 2013, he has a research grant at the Department of Social, Political and Cognitive Science of the University of Siena and is Ph.D. candidate at the Department of Electronics and Telecommunications of the University of Florence. His interests include different design fields like fashion design, product design and interaction design. His works are inspired by the theme of nature and its phenomena, interpreted as source of "systemic" and aesthetic inspirations.

E-mail: michele [dot] tittarelli [at] unisi [dot] it

Nicholas True obtained a Master's degree in human-computer interaction from the $\mathrm{HCI} / \mathrm{d}$ program at the School of Informatics and Computing at Indiana University. Currently, he is a Ph.D. candidate in the Department of Informatics at Umeå University in Sweden.

E-mail: nicholas [dot] true [at] informatik [dot] umu [dot] se

Nigel Papworth studied graphic design at the London College of Printing, graduating in 1982 with a specialisation in handmade printing techniques. After graduation, he worked as a designer and illustrator in London until 1985, when he moved to Umeå, Sweden. After working in advertising in Sweden for a number of years, Nigel co-founded one of Sweden's first major computer games companies, Daydream Software, in 1994. After 10 years and a number of successful games, on which he worked as principle designer, Nigel left Daydream to pursue a more research oriented vision on interactivity and games. His main area of interest is the study of user response to interactive media and how the design process can make this more intuitive and interesting. His current work investigates new methodologies in regard to artificial intelligence, user perception and the utilisation of unusual interface solutions.

E-mail: nigel [at] tii [dot] se

Caroline Hummels is full-professor 'Design and Theory for Transformative Qualities' in the Department of Industrial Design at Eindhoven University of Technology. Together with Prof. Ron Wakkary, she heads the Designing Quality in Interaction group at Eindhoven University of Technology. Furthermore, she leads, together with Prof. Peter de With, the interdepartmental theme Participatory Health \& Well-being at the same university. In her research, she develops frameworks, tools and concepts to support designing toward transformation. Her work addresses aesthetic interaction, craftsmanship, skills, direct perception theories, health and well-being, multi-stakeholder cooperation and innovation. E-mail: c [dot] c [dot] m [dot] hummels [at] tue [dot] $\mathrm{nl}$ 


\section{Acknowledgments}

There are many people who actively contributed to the exhibition and that we sincerely thank for their precious support: Fabio Celli (poems curator), Fabio Pianigiani and Narada Studio (music and recordings), Albalisa Sampieri, Persone Libro di Siena - Associazione Donne di Carta (Living

Books Association), Dominik Legault, Emily Sample, Susan Braden, Jenny Chan Yu Ching (voices). We are particularly grateful to Stoffel Kuenen, Fredrik Nilbring and Björn Yttergren who were indispensible in building the installation, Iolanda Iacono who contributed to the management of the exhibition, Ernesto Di Iorio and QuestIt who provided the software WhatsOn, Matteo Sirizzotti and Lorenzo Megale who set up the Backstage, Max Pardeilhan who helped during the construction and de-construction of the installation, and finally, the Umeå Studio of Interactive Institute Swedish ICT.

\section{Notes}

1. Foka and Arvidsson, 2014, p. 1.

2. A selection of poems were recorded and played in both their native Italian, as well as translated versions in English. The poems were: $\mathrm{E}$. Sanguineti, "Ballata delle donne," in Ballate (1982-1989); S. Plath, "Canzone della donna perduta," in Tutte le poesie (1956); S. Plath, "Lifting," in Tutte le poesie (1961); W. Szymborska, "La gioia di scrivere," in Uno spasso (1967); W. Szymborska, "Vietnam," in Uno spasso (1967). W. Szymborska, "Ritratto di donna," in Grande numero (1976); W. Szymborska, "Torture," in Gente sul ponte (1986); A. Merini, "Ape Regina," in Vuoto d'amore (1987-1990); A. Rosselli, "Impromptu," in Impromptu (parte 13) (1981/82); A. Rosselli, "A me stessa," in Palermo (1963, Primi scritti); and M. Luzi, "Le donne di Bagdad," in Appendice 'Sia detto' (1991).

\section{References}

M. Birenbaum, 2003. "New insights into learning and teaching and their implications for assessment," In: M. Segers, F. Dochy and E. Cascallar (editors). Optimising new modes of assessment: In search of qualities and standards. Dordrecht: Kluwer, pp. 13-36.

J. Dewey, 1933. How we think: A restatement of the relation of reflective thinking to the educative process. Chicago: Henry Regnery.

P. Dourish, 2001. Where the action is: The foundations of embodied interaction. Cambridge, Mass.: MIT Press.

Anna Foka and Viktor Arvidsson, 2014. "Digital gender: A manifesto Report on the Research Workshop Digital Gender, Theory, Methodology, and Practice" (15 May), at http://ssrn.com/abstract=2437659, accessed 26 March 2015.

doi: http://dx.doi.org/10.2139/ssrn.2437659, accessed 26 March 2015.

P. Marti and C.J. Overbeeke, 2011. "Designing complexity in context: Light through Culture," CHItaly: Proceedings of the Ninth ACM SIGCHI Italian Chapter International Conference on Computer-Human Interaction: Facing Complexity, pp. 65-70.

A. Pollard, 2005. Reflective teaching: Evidence-informed professional practice. Second edition. London: Continuum.

D. Schön, 1983. The reflective practitioner: How professionals think in action. New York: Basic Books.

R. Sennett, 2008. The craftsman. London, Penguin.

\section{Editorial history}

Received 3 March 2015; accepted 24 March 2015.

Copyright (c) 2015, First Monday.

Copyright (c) 2015, Patrizia Marti, Jeroen Peeters, Ambra Trotto, Michele Tittarelli, Nicholas True, Nigel Papworth, and Caroline Hummels. All Rights Reserved.

Embodying culture: Interactive installation on women's rights by Patrizia Marti, Jeroen Peeters, Ambra Trotto, Michele Tittarelli, Nicholas True, Nigel Papworth, and Caroline Hummels.

First Monday, Volume 20, Number 4 - 6 April 2015

http://firstmonday.org/ojs/index.php/fm/article/view/5897/4418

doi: http://dx.doi.org/10.5210/fm.v20i4.5897 
A Great Cities Initiative of the University of Illinois at Chicago University Library.

(c) First Monday, 1995-2015. 